\title{
Energy Efficient Data Dissemination in Multi-UAV Coordinated Wireless Sensor Networks
}

\author{
Vishal Sharma, ${ }^{1}$ Ilsun You, ${ }^{2}$ and Rajesh Kumar ${ }^{1}$ \\ ${ }^{1}$ Computer Science and Engineering Department, Thapar University, Patiala, Punjab 147004, India \\ ${ }^{2}$ Department of Information Security Engineering, Soonchunhyang University, Asan-si 31538, Republic of Korea
}

Correspondence should be addressed to Ilsun You; ilsunu@gmail.com

Received 29 March 2016; Accepted 4 May 2016

Academic Editor: Daniel G. Reina

Copyright (C) 2016 Vishal Sharma et al. This is an open access article distributed under the Creative Commons Attribution License, which permits unrestricted use, distribution, and reproduction in any medium, provided the original work is properly cited.

With the requirement of better connectivity and enhanced coverage, collaborative networks are gaining a lot of popularity these days. One of such collaborative networks is formed between the wireless sensor networks (WSNs) and the unmanned aerial vehicles (UAVs). WSNs comprise static nodes arranged in a flat grid topology which may be randomly deployed or by some particular distribution. With the sensors operating on batteries, WSNs face a crucial issue of energy depletion during network operations. Integration of WSNs with UAVs can provide a solution to this excessive utilization of energy resources. UAVs provide a maneuvering support by playing a pivotal role of a manager node in these networks. However, integration of these networks demands an improved approach for data dissemination for effective utilization of network resources. For this, we propose a new data dissemination approach, which utilizes the attraction properties of fire fly optimization algorithm to provide energy efficient relaying. The proposed approach provides continuous connectivity, better lifetime, and improved coverage in the UAV coordinated WSNs. The performance of the proposed model is presented in terms of significant gains attained for parameters, namely, throughput, coverage, mean hops, lifetime, and delays, in comparison with the EEGA, ERIDSR, and I-ERIDSR approaches.

\section{Introduction}

Collaborative networks help resolving tasks that are relatively complex to be attained using a single network model. One of the collaborative networks can be formed using the unmanned aerial vehicles (UAVs) and the wireless sensor networks (WSNs). These networks can provide support in various applications concerning tracking and data acquisition problems. UAVs have tendency to fly autonomously and can collaborate with existing network to form a search, tracking, and data acquisition networks [1]. Further, these networks can be used to provide vast coverage and enhanced security in border areas [2]. These vehicles are capable of forming intermittent connections with the existing wireless networks to form fully reliable opportunistic networks that can sort issues like broadcast storm, network partitioning, and so forth [3]. With the tendency to organize and resolve the failure issues easily, these vehicles can be used to form selfsufficient networks that can withstand the sudden network changes and failures [4].
In alteration to the UAVs, WSNs are comprised of sensor motes that are arranged using grid-like formation, random deployment, or distribution. Motes in sensor network are static devices that operate over the battery and provide highly sensitive applications in data acquisitions $[5,6]$. WSNs can be used in remote areas where direct data acquisition is not in the reach of humans. Traditional WSNs follow the topology comprising of base station, manager nodes, and the numerous sensor motes. The topology may vary from application to application, but architecture remains the same. Excessive consumption of energy and optimal route selection in WSNs are still an open issue that requires an optimal solution to form an efficient WSN [7-9].

The collaboration between the UAVs and the WSNs can provide a vast range of applications such as sensor flocking, border surveillance, area monitoring, remote data gathering, and obstacle avoidance [6,9-11]. Collaborative network between the UAVs and the WSNs can provide an efficient solution to the routing loop problem in traditional sensor networks and can also enhance the lifetime of the sensors 
by optimal division of the load. However, these solutions are subjected to the formation of an optimal topology and a routing schema for utilization of the coordination between the UAVs and the WSNs. Also, the positioning of UAVs in sensor networks is an optimization problem that needs to be resolved for efficient deployment of such collaborative network models.

One possible solution to these issues is presented in this paper that aims to organize the coordinating sensor networks and the UAVs into a series of segments as done in the traditional WSNs. However, the proposed model differs in the architectural implementation as it uses UAVs as manager nodes instead of keeping static nodes as managers. Further, segments serve as the waypoints for the UAVs. The data forwarding in the proposed collaborative network is performed using the properties of fire fly optimization algorithm (FFOA) [12].

FFOA is the nature-inspired optimization algorithm that derives its properties from the fire flies that have tendency to attract each other based on their light intensity. The existing optimization function is modified to make it suitable for the proposed network model. Then, the data dissemination is proposed in terms of distributed algorithms by considering the interactions between the different types of nodes in these collaborative networks. FFOA is preferred over other algorithms because of similarity in the energy concept of WSNs and the light intensity based attraction property of the fire flies. Further, the convergence rate of the FFOA in case of multiobjective problems is comparatively faster than any other nature-inspired algorithm [12].

The remaining part of the paper is organized as follows: Section 2 presents a brief description of the problem and our contribution in resolving it. Section 3 presents the related work. Section 4 presents the proposed approach comprising the network deployment, energy mode, routing model, and data dissemination between the UAVs and the WSNs. Section 5 evaluates the performance of the proposed approach in comparison with the existing approaches. Finally, Section 6 concludes the paper.

\section{Problem Formulation and Our Contribution}

Wireless sensor networks face a critical issue of energy depletion during their prolonged activity. Limited availability and continuation of decaying of available energy increase the chances of occurrence of the dead state. Dead state refers to the state in the transmission procedure when the node is unable to receive, process, and transmit the data. Further, the existing routing protocols and data dissemination approaches on WSNs and UAV coordinated WSNs suffer from an issue of routing loop. With long range deploying of sensor motes, the probability of occurrence of routing loop increases as the motes are unable to route the data from their segment to the motes in the other segment. This leads to fast wastage of energy resources and increased delays in highly critical data gathering environments.

Also, the interfacing between the UAVs and the sensor nodes raises the three coordination aspects to be handled for data transmission towards the base node, namely, UAV to UAV, UAV to sensor nodes, and sensor to sensor coordination. In this paper, all these problems have been clearly identified, and a novel solution is proposed using fire fly optimization algorithm. The proposed fire fly based energy efficient data dissemination approach is capable of providing continuous, low-complex, and highly stable approach, which is capable of enhancing the lifetime of the existing WSNs.

\section{Related Work}

Integration of traditional WSNs with UAVs provides various applications, particularly focusing on the energy efficient data acquisition. Using aerial nodes as intermediates in the existing networks can resolve various existing issues, such as network loop, routing loop, and network partitioning. One of the utilities of the WSNs in collaboration with the UAVs is given by Faiçal et al. [13]. The authors focus on forming optimal routes for control and spraying of pesticides on the crop field using a predefined topology. The paper provides a novel data acquisition approach in agricultural applications.

A division of the area for optimal placement of the sensors can also provide an optimal routing solution for efficient data dissemination in WSNs. Some of these approaches include energy efficient routing using relative identification and direction (RIDSR) developed by Weng and Lai [14] which is based on the RDSR protocol developed by Oh et al. [15]. The proposed protocol divides the area into a series of octagons and resolves the issue of routing loop in WSNs by maintaining the energy of the network above a certain threshold value. In an extension to this protocol, Wei et al. [16] further optimize the RIDSR for its load by redefining node arrangement and communication cost. However, these protocols are not suitable for collaborative WSNs as these may lead to routing loop because of varying topology of the aerial nodes.

Use of genetic algorithms can be an alternate solution in the formation of energy efficient WSNs. A multisink based sensor network model is utilized by Safa et al. [17] for the development of an energy efficient genetic approach for data dissemination in WSNs. Fitness functions are derived from the selection of the next hop. This approach can be tested over UAV-like infrastructures, and defining a new fitness function with less complexity can provide a support for UAV to sensor communications.

Energy depletion at regular intervals despite of low connectivity is one the major issues faced by the sensor networks. Zhang et al. [18] developed a new algorithm that is capable of balancing the energy in the network. The algorithm developed by the authors uses a link cost function formed by a combination of various parameters such as residual energy and load factors to select the optimal route between the nodes. De Freitas et al. [19] designed a decentralized UAV oriented sensor network for surveillance. The approach developed by the authors uses static sensors that make alarms indicating the presence of possible targets to be handled by the UAVs.

Abdelhakim et al. [20] proposed a mobile access based wireless sensor network to resolve the issue of limited speed 
at the physical layer. The authors have proposed a new topological arrangement for the nodes that is able to provide enhanced throughput and coverage with lesser delays. Border surveillance is the other application of the UAV oriented WSNs developed by Berrahal et al. [2]. This approach provides a novel solution for optimal placement of the sensors in the border areas. Further, the proposed approach provides energy efficient techniques to accurately map the terrestrial sensors using RFID technology.

Another problem with the UAV coordinated WSNs is the sensor localization. Valuiskyi et al. [21] presented this problem of sensor localization using UAVs. Selection of the rational topology and network controller is the primary focus in their discussions. Further, distributed greedy algorithms can provide delay sensitive data forwarding in traditional sensor networks. One of such approaches is given by $\mathrm{Li}$ et al. [22]. First and second forwarding nodes schemes are used for data forwarding by operating them over the greedy distributed algorithms.

The approaches developed so far are capable of handling data efficiently either in application oriented UAV coordinated WSNs or in traditional WSNs only. However, a generic dissemination approach is required that not only resolves the routing loop issues in WSNs but also provides an energy efficient data forwarding approach with effective load balancing and connectivity.

\section{Proposed Approach: Energy Efficient Data Dissemination}

The proposed approach aims at improving the topological as well as the data dissemination issues in traditional WSNs by the use of multiple UAVs. These UAVs are capable of providing direct connectivity between the sensors and the base station. Also, these UAVs act as a pivot between the existing sensor nodes that can regulate the traffic flow efficiently. The proposed approach also eradicates the possibilities of routing loop problem that may arise in traditional WSNs during long range of deployment. The proposed approach uses the fire fly optimization algorithm (FFOA) as a base for connectivity between the network nodes. FFOA provides support for using the attraction properties of fire fly to identify the network nodes for coordination. The whole network is divided into three major coordination aspects, namely, UAV to UAV, UAV to sensors, and sensors to sensors. Using FFOA, three different attraction based objective functions are derived for these coordinations. The proposed approach first presents the system model that provides the insight of the energy model considered in the proposed network model, and then it uses this model to form a data dissemination algorithm for improved and prolonged connectivity.

4.1. Network Deployment. The network comprises three types of nodes, namely, the base node, sensor nodes/motes, and the UAVs. The UAVs considered in the proposed model have the capability of flying autonomously with possible maneuvers according to $\Theta \in(L, R, F, B) \pm 45^{\circ}$. The whole area is divided into regular circular segments encircling a square as

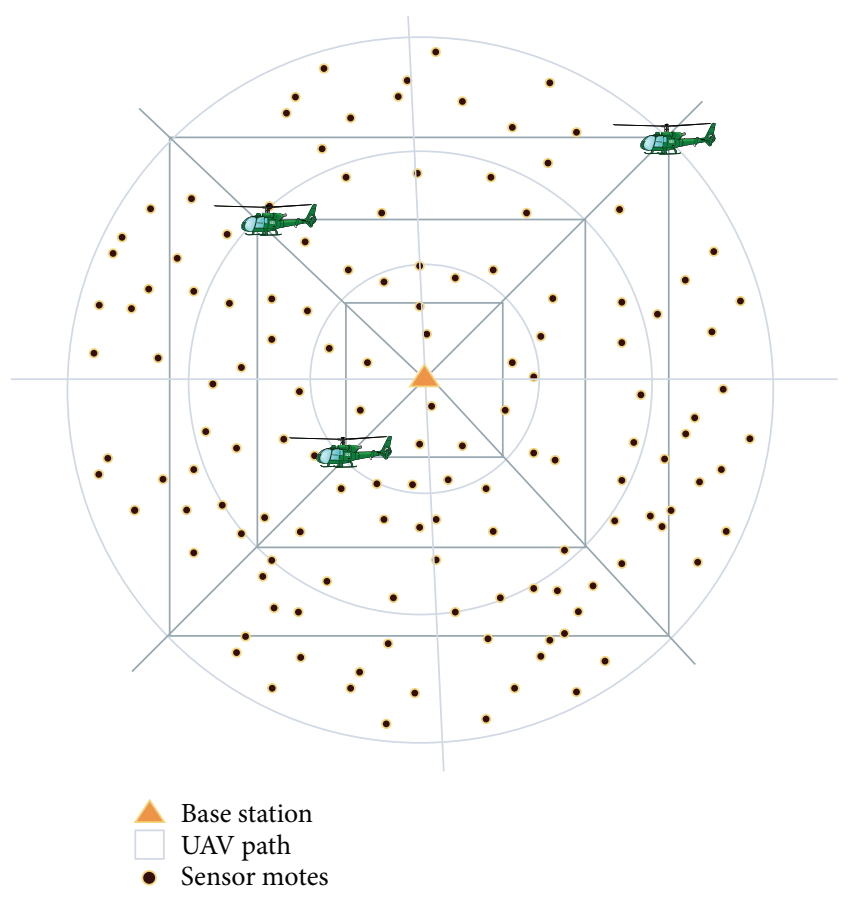

FIGURE 1: Network deployment for UAV coordinated WSNs.

its inner polygon that also forms the pathway for the UAVs. Number of UAVs is set equal to the number of segments. The sensor motes can be placed in a grid, random deployment, or according to some distribution. In the proposed model, the sensor motes are deployed using Poisson Distribution as shown in Figure 1.

Further, based on the network deployment, three coordinations are possible for data dissemination, namely, UAV to UAV, UAV to sensors, and sensors to sensors. This coordination forms the backbone of the proposed approach and helps in developing an algorithm that can be deployed as independent threads to each of the abovementioned coordinations. These three network coordinations for data dissemination are shown in Figures 2, 3, and 4.

4.2. System Model. The system comprises base station surrounded by $m$ number of sensor nodes, maneuvered by $n$ number of UAVs. Here, $n$ is computed based on the number of segments into which the areas around base station are divided. The energy model of the proposed approach aims at the formation of the attraction based objective function that drives the data dissemination in these coordinated networks. Let $\mathrm{PC}_{i}$ be the power consumption of the $i$ th sensor, where $i \in S$. Now, the energy utilization $\mathrm{EU}_{i}$ of the $i$ th sensor for time $t$ is given as

$$
\mathrm{EU}_{i}=\mathrm{PC}_{i} \times t
$$

where, considering a unit power consumption for each of the message,

$$
\mathrm{PC}_{i}=\frac{M_{s} \times D_{c}}{C_{h}} .
$$




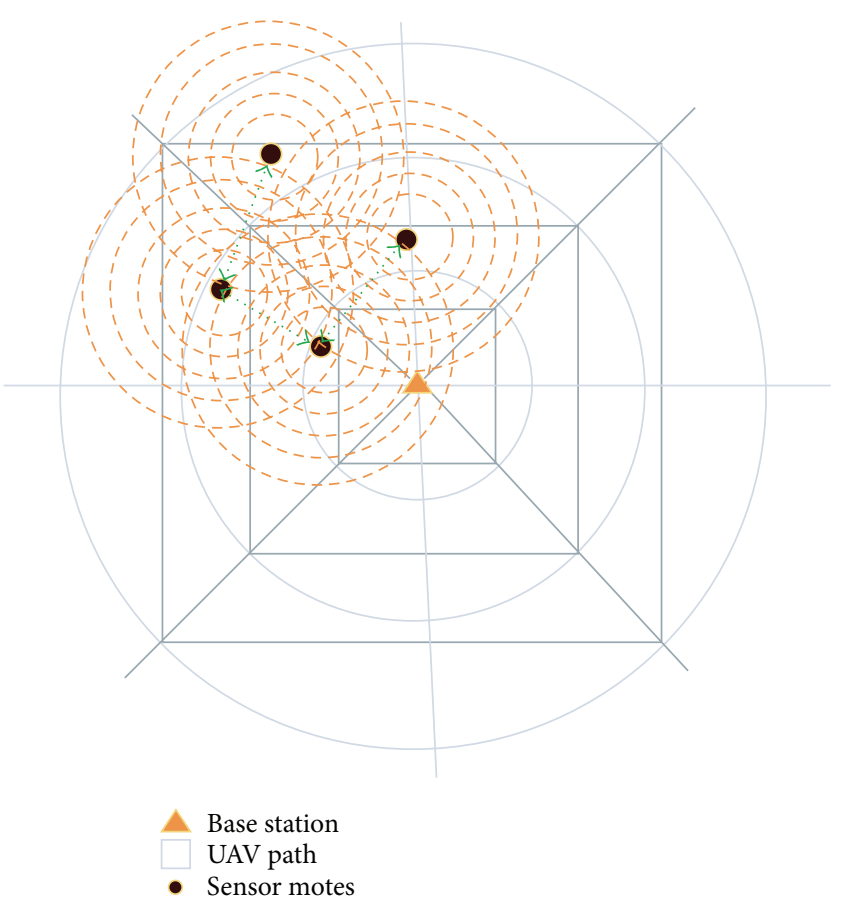

FIGURE 2: Sensors to sensors mapping.

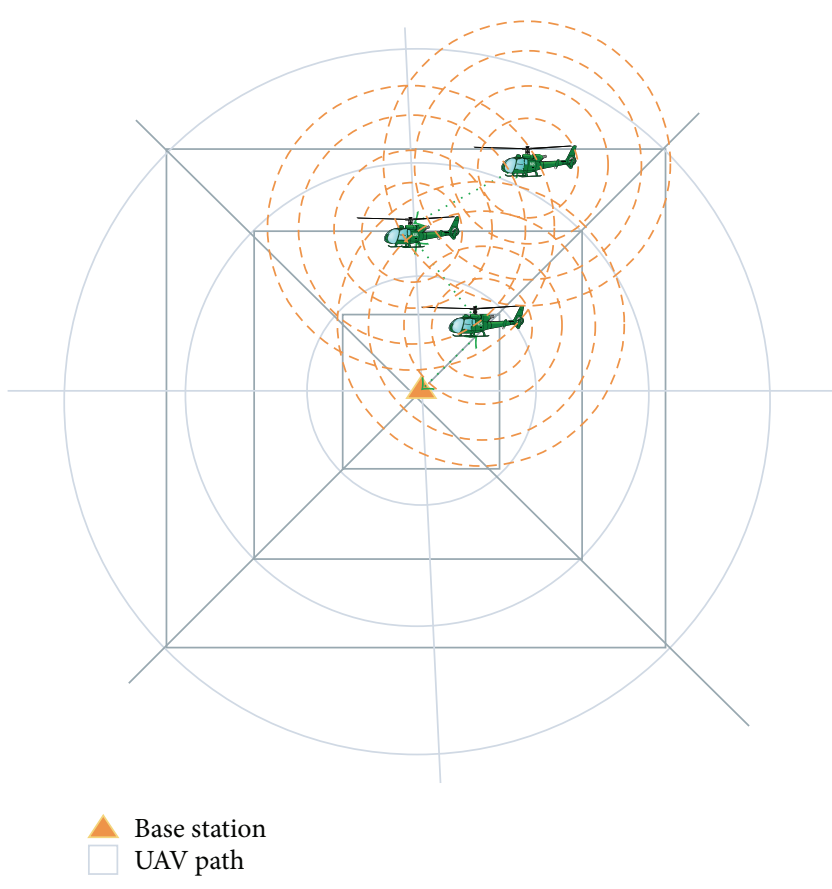

FIgURE 3: UAV to UAV mapping.

Here, $M_{s}$ are the number of messages processed over $C_{h}$ number of channels with $D_{c}$ degree of connectivity. Using this model, energy consumption $\mathrm{EU}_{a}$ for the overall areas with $S$ number of segments is given as

$$
\mathrm{EU}_{a}=\sum_{j=1}^{S}\left(\sum_{i=1}^{K} \mathrm{PC}_{i}\right) \times t,
$$

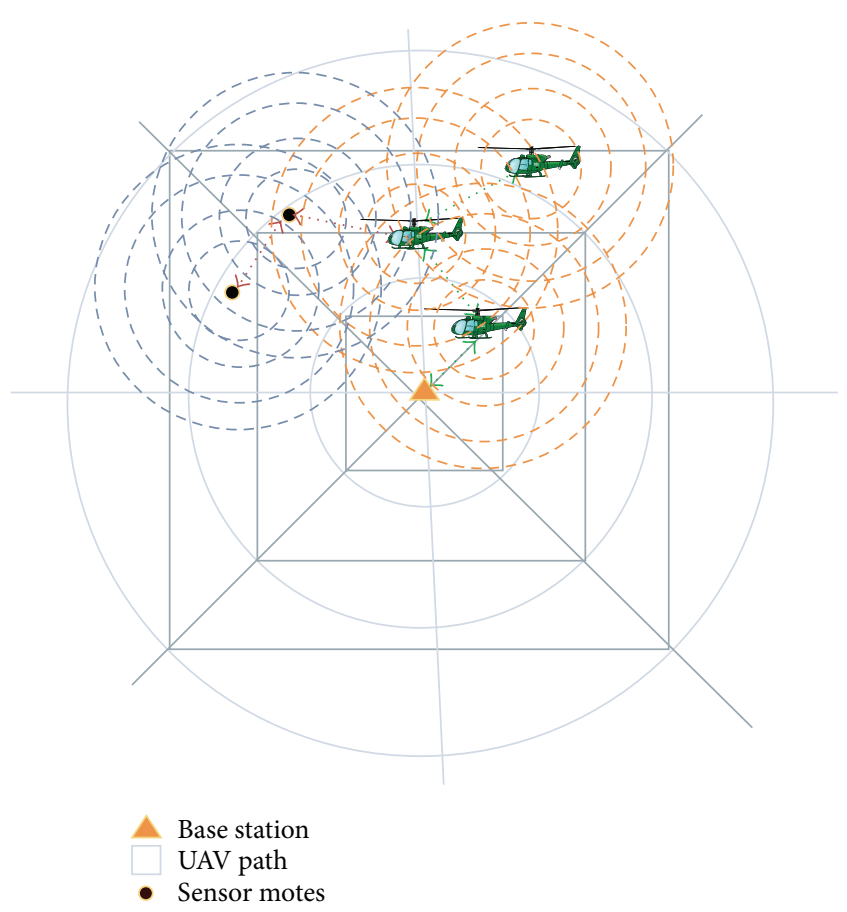

FIGURE 4: Sensors to UAV mapping.

where $K$ is the number of sensors in Sth segment such that

$$
\sum_{i=1}^{S}(K)_{i}=m
$$

For the overall network, the depleted energy $E_{d}$ is given as

$$
E_{d}=\frac{E_{\mathrm{TX}}+E_{P}}{\text { load }} .
$$

Here, load is variant in terms of coordination, namely, UAV to UAV, UAV to sensors, and sensors to sensors.

4.2.1. Sensors to UAVs Coordination. For sensors to UAVs, the load over UAV is calculated over the energy model as

$$
\operatorname{load}_{s u}=\int_{y \in A} \frac{U_{K} \times D_{r}}{S_{\max }} d y,
$$

where $y$ is the location of a UAV in the segment $U, U_{K}$ is the number of sensors in the segment $U$ handled by the UAV, $D_{r}$ is the depletion rate of the UAV energy, and $S_{\max }$ is the maximum sensors accommodated by the UAV. The primary focus of the UAV to sensors coordination is to prevent the excessive energy depletion for UAVs and load balancing that will eventually prevent UAVs from being in a dead state. This load balancing is attained using the route rehabilitation and maintenance during recovery phase. For $\eta$ number of active sensors interacting with the UAVs at any time instance, the energy depletion rate is considered to follow the decay rule of $e^{-\eta}$. Further, following this decay rule, for continuous transmission, the left out energy over each UAV should not be less than $e^{-\eta} / U_{K}$. If the rate of depletion goes beyond this value, the possibility of UAV to be in dead state increases; that may result in the loss of an aerial vehicle. 
4.2.2. UAV to UAV Coordination. For UAV to UAV communication, load over UAV is computed as

$$
\operatorname{load}_{u}=\int_{y \in K} \frac{\mu_{a} \times \mu_{s}}{C_{p}} d y,
$$

where $\mu_{a}$ is the packet arrival rate, $\mu_{s}$ is the packet size, and $C_{p}$ is the channel capacity. This load is computed for a UAV currently at a location $y$ in the segment $K$. The depletion rate is considered to be similar as that of UAVs. The UAVs are considered to be operating over a dedicated band with finite link capacity.

4.2.3. Sensors to Sensors Coordination. For sensors to sensors coordination, the load over each sensor is calculated over the energy model as

$$
\operatorname{load}_{s}=\int_{x \in A} \frac{D_{s} \times D_{r}}{U_{\max }} d y,
$$

where $D_{s}$ is the degree of connectivity for sensor at location $x$ in the area $A$ and $U_{\max }$ is the maximum number of UAVs a sensor can interact during same time instance. The depletion rate $D_{r}$ for the sensor to sensor connectivity is computed as $e^{-\eta_{D_{s}}}$.

The left out energy at any instance is denoted as $E_{L}$ (= EU $-D_{r}$ ), which should always be greater than the total energy required for network operations $E_{\text {total }}\left(=E_{\mathrm{TX}}+E_{P}\right)$. However, there might be cases when some of the sensors are on the verge of the dead state while others are having remaining energy that is way too high than the other sensors within the same segment. Here, UAV s come out to be handy; that is, UAVs with the capability of forming autonomous networks will form sink with the base and source with the sensors possessing an optimal amount of energy provided that the properties of FFOA are maintained. Also, for the whole network, the deviation in the energy of each node with respect to the mean network energy should always be greater than the energy per load, that is,

$$
\sqrt{\frac{1}{m} \sum_{i=1}^{m}\left(\mathrm{EU}_{i}-\overline{\mathrm{EU}}\right)^{2}} \geq E_{\mathrm{total}}
$$

where load is additive of load $\operatorname{load}_{u}$, and $\operatorname{load}_{s}$.

4.3. Attractiveness Using Fire Fly Optimization. The attractiveness is the property of the fire flies by which they attract each other because of common gender [12]. The optimization function for attractiveness is modified for its applicability in selection of new routes in sensors to sensors, UAV to sensors, and UAV to UAV coordination. The function is similar for the UAV to UAV and UAVs to sensors coordination but changes for sensors to sensors where pathloss is not considered as an active variable because of fixed topology. Now for UAV to UAV and sensor to sensor coordination, the attractiveness over the node $i$ with respect to the node $j$ is calculated using a fire fly optimization function as

$$
\text { node }_{i}=E_{L, i}+\mathrm{EU}_{0} e^{-D_{r} \rho^{2}}\left(\text { node }_{i}-\text { node }_{j}\right)+P(\lambda),
$$

where $E_{L, i}$ is the left out energy, $\rho$ is the average pathloss, $\left(\right.$ node $_{i}-$ node $_{j}$ ) is the distance between the two nodes, $\mathrm{EU}_{0}$ is the initial energy, and $P(\lambda)$ denotes the vector for distribution of nodes using Poisson Distribution such that

$$
\lambda= \begin{cases}n, & \text { Case } 0 \text { UAV to UAV mapping } \\ n+m, & \text { Case } 1 \text { UAV s to sensors mapping. }\end{cases}
$$

Now, for sensors to sensors mapping, this objective function changes to

$$
\begin{aligned}
\operatorname{node}_{i}= & E_{L, i}+\mathrm{EU}_{0} e^{-D_{r} r^{2}}\left(\operatorname{mode}_{i}-\text { node }_{j}\right) \\
& +P(\lambda=m)
\end{aligned}
$$

where $r$ is the average radio range of the sensor node. From (11) and (12), the attraction for each node in UAVs to sensors and UAV to UAV mapping is computed as

$$
A_{\text {us }} \text { or } A_{u u}=\mathrm{EU}_{0} e^{-D_{r} \rho^{2}} \text {, }
$$

and for sensors to sensors is given by

$$
A_{s s}=\mathrm{EU}_{0} e^{-D_{r} r^{2}} \text {. }
$$

This value of attraction operates over certain threshold value to resolve the routing loop problem during route selection between the base station and the sensor nodes. This threshold value is determined based on the segment between which the data is to be transmitted. According to this principle, the deviation between the attraction of the source segment should be less than the deviation of the destination segment, that is,

$$
\begin{aligned}
& \left(\sqrt{\frac{1}{U_{K}} \sum_{i=1}^{U_{K}}\left(A_{i}-\bar{A}\right)^{2}}\right)_{\text {source }} \\
& \leq\left(\sqrt{\frac{1}{U_{K}} \sum_{i=1}^{U_{K}}\left(A_{i}-\bar{A}\right)^{2}}\right)_{\text {sink }} .
\end{aligned}
$$

4.4. Route Formation and Data Forwarding. As classified above, the routing is subjected to three major possibilities based on the network deployment. With variation in attraction properties, the nodes with more provisioning of remaining energy are used for routing. This left out energy takes into account the decay rule over the radio range and the distance. Route selection is performed by selecting the next optimal node from other segments. UAVs in the proposed approach act as manager nodes; thus, the primary task of the sensors is to forward the data to the nearest aerial node which also posseses a high attraction value. Then, these aerial nodes decide the routes towards the base station. This routing is based upon the trajectory of the aerial nodes and their radio range. Three main algorithms are used for data dissemination in sensors to sensors coordination, sensors for 
(1) Input: $m, K, A_{s s}$

(2) set Flag $=0$

(3) while (transmission != end) do

(4) mark the source $(\in m)$

(5) broadcast "Hello" $\leftarrow A_{s s}$

(6) $i=$ segment of current source $(\epsilon K)$

(7) while (next hop found != true) do

(8) sort nodes in order of their attraction

(9) select upper node and check for its connections

(10) if (connections available $==$ true) then

(11) continue

(12) else

(13) select next optimal node

(14) end if

(15) check for satisfaction of (9) and (15)

(16) if (satisifaction $==$ true) then

(17) select node as next hop

(18) $\quad$ Flag $=0$

(19) else

(20) $\quad$ Flag $=1$

(21) end if

(22) end while

(23) if (flag $==0$ ) then

(24) transmit()

(25) else

(26) check for nodes from same segment and perform steps (8)-(15)

(27) end if

(28) iterate till data is not completely transmitted

(29) end while

Algorithm 1: Data dissemination: sensors to sensors.

UAV s coordination, and UAV to UAV coordination, as shown in Algorithms 1, 2, and 3, respectively.

Algorithm 1 is used in case of sensors to sensors routing. These algorithms account for the attraction properties of the sensors derived on the basis of properties of the fire fly algorithm. In the initial run, the algorithm takes into account the source selected from the region of interest (ROI) of sensors. The whole network model makes announcements regarding the attraction value of nodes using HELLO messages. Then, in the next step, nodes are arranged in order of their attraction value, providing that the conditions in (9) and (15) are satisfied by the node which is to be selected next. Further, during implementation of this algorithm, it is also taken into account that the next hop is not selected from the same segment. However, there might be cases when no node is present in the next segment with a higher attraction value. In that case, the nodes from the same segment are considered till the availability of a node from the next segment provided that the node of the same segment has not been used earlier for routing during the current iteration.

Algorithm 2 is used for sensors to UAV marking and data forwarding. The algorithm operates till all the broadcast messages in the network vanish indicating no further requirement of routing. In the initial conditions, the algorithm marks the sensors that have data to be transmitted to the base station. Since UAVs are acting as the manager nodes, this procedure requires selection of optimal and efficient UAV for accurate and faster communication between the source and the sink. All the UAVs are also sorted in order of their attraction properties and the one with the best value gets selected for transmission. UAVs are also bound to satisfy (9) and (15). There might be cases when more than two UAVs are available with same attraction value; in that case, the one closest to the base station is selected for data forwarding.

Algorithm 3 provides support for the UAV to UAV routing. The selection of the UAVs is marked by the attraction property similar to sensors to sensors routing. The algorithms operate with different conditions depending upon the number of UAVs assigned to each segment. Although in the proposed approach the number of UAVs is kept constant with a count of one per segment, yet the algorithm is scalable and can be used for any number of UAVs in a particular segment. Figure 5 presents the flow chart for the proposed data dissemination approach.

4.5. Routing Loop Problem. With the network node selected on the principle of the attraction property of the fire fly, the value of attraction changes after each iteration, thus eradicating the chance of selection of similar node that may cause the loop in the network. A routing spanning tree comprising the nodes with maximum attraction is formed which primarily selects the nodes from the different segments, thus advancing 


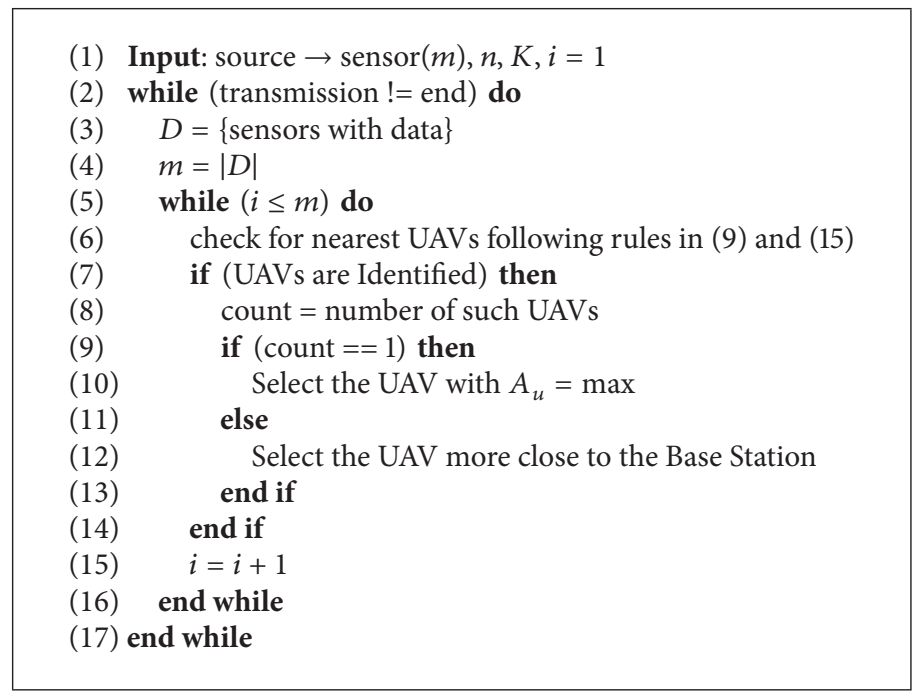

Algorithm 2: Data dissemination: sensors to UAVs.

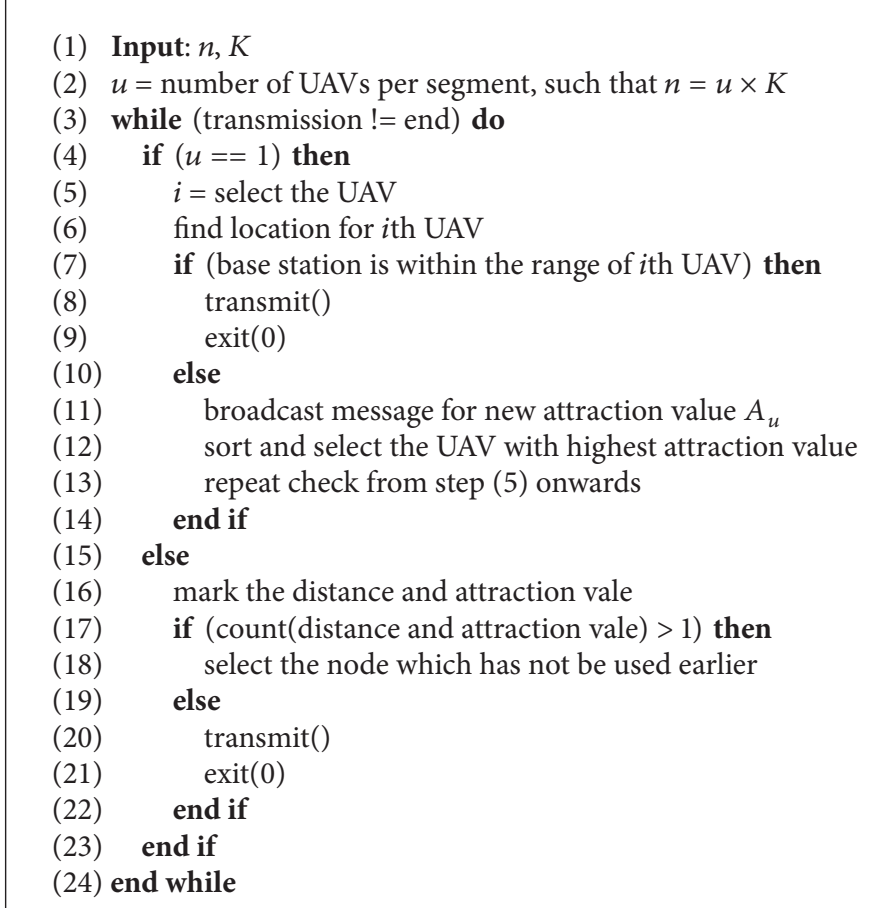

Algorithm 3: Data dissemination: UAV to UAV.

data towards the base station. Further, in the case of nodes belonging to the same segment, selection of nonrepetitive nodes despite being the second highest in attraction value provides a nonredundant node marking for energy efficient data dissemination. However, this selection is subjected to the constraints defined in (9) and (15).

Also, the proposed approach optimally selects the nodes using an attraction based objective function, which, provides a low-compelx, and more stable data forwarding with nonredundant node selection and higher accuracy with the provisioning of load balancing.

4.6. Route Rehabilitation and Maintenance. There is always a possibility of the nodes running out of energy at the same time instance due to prolonged connectivity. This energy loss should be regular such that the load is optimally balanced between the nodes of the ROI. Further, the path may encounter false routes because of the nonoverwriting initial 


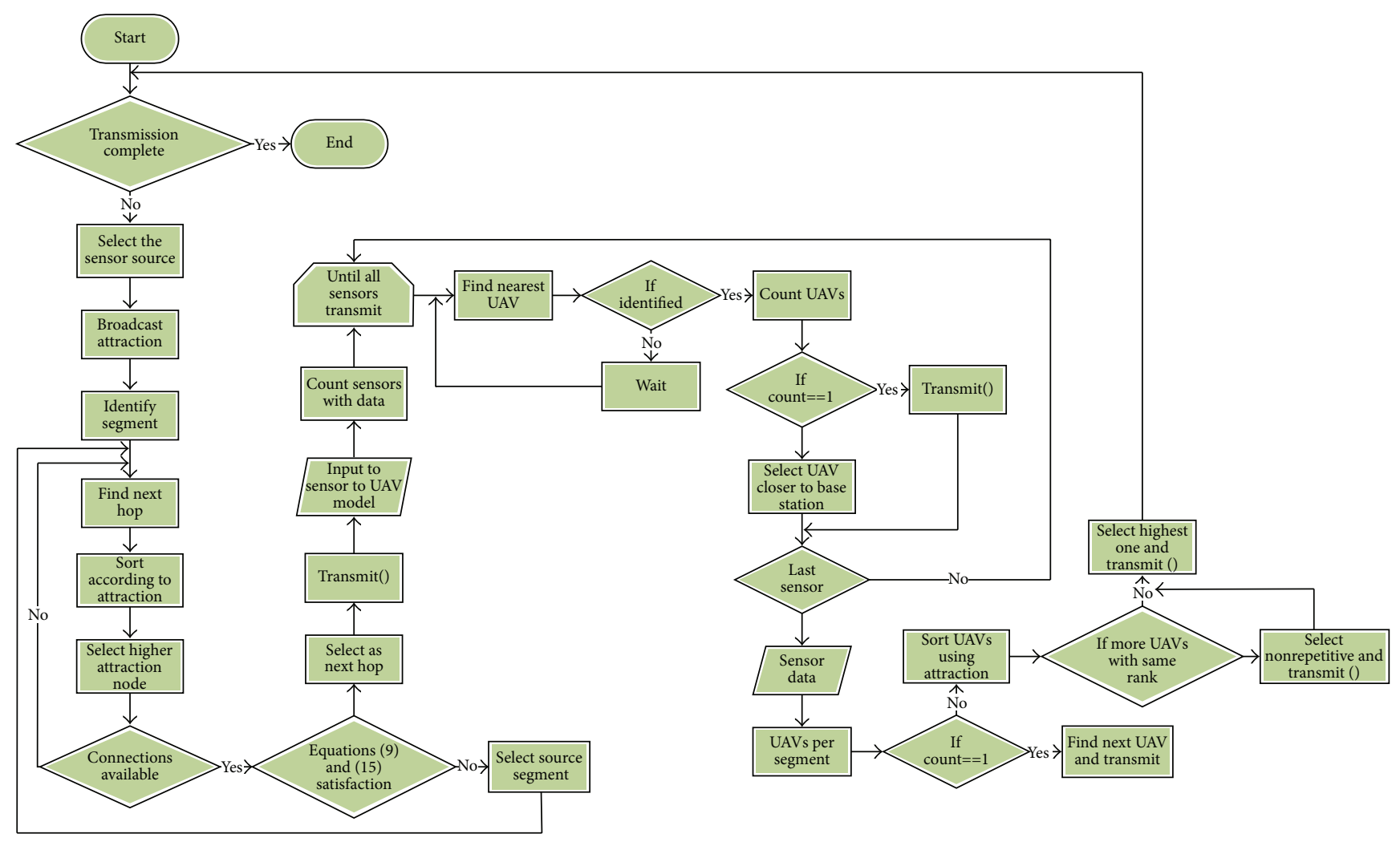

FIGURE 5: Flowchart: energy efficient data dissemination.

broadcast messages. In such cases of false routes in the network, route rehabilitation and continuous maintenance are used to form the continuous and robust data dissemination approach.

For route rehabilitation and maintenance, Algorithm 4 provides the pseudocode that can effectively resolve the node failure and dead state issues without causing any loop. The proposed algorithm initially tries to make reannouncements for nonresponding nodes; upon success, the route is revived. However, in case of failures and unavailability of nodes with high energy, the priority order of the requests is changed to the current request, and the number of in-degree and out-degree is reduced so as to allow the new requests to be handled at once, thus, providing the provision of route maintenance without consumption of much energy.

\section{Performance Evaluation}

The proposed energy efficient data dissemination approach of UAV coordinated WSNs uses the attraction property of the existing fire fly optimization algorithm. The proposed model was evaluated over an area containing a base station, UAVs as manager node, and sensors deployed using the Poisson Distribution over ROI of $50 \times 50 \mathrm{~m}^{2}$. The initial energy of the sensors is considered to be 150 Joule (J), and for UAVs, the energy is assumed to be far more than the requirement of the whole network. However, for simplicity of the simulations, the initial energy of the UAVs is $2000 \mathrm{~J}$.
The number of segments varied from 3 to 5 per base station, so is the number of UAVs. Total number of sensors varied between 100 and 300. Using the standard model, the energy consumption per sensor was $0.5 \mathrm{~J}$. The radio range of the sensors is between $10 \mathrm{~m}$, and that of the UAV is $500 \mathrm{~m}$ radially. The other parameters configured for performing comparative analysis of the proposed approach are shown in Table 1. The taxonomy of the various parameters used for the analysis of the proposed approach are as follows:

(i) Throughput: in the proposed model, UAVs play an important role in pivoting messages between the base station and the sensor nodes. This metric accounts for the actual load offered by the network over the connected channels. Further, throughput also accounts for the traffic rate of the complete network. Higher value of throughput accounts for better network performance. Also, an optimal rate should be maintained in the network which prevents overconsumption of energy.

(ii) Mean Hops: the more the number of intermediate nodes, the more the energy consumption. The proposed approach, focuses on the selection of the node from the next segment towards the UAV or the base station. This metric accounts for analysis of the actual number of intermediates used in an average simulation run of the network model.

(iii) Packet Delivery Ratio: successful transmission allows better relaying. The packet delivery ratio is the actual 
(1) Input: current routing state

(2) while (transmission != end) do

(3) check for next hop

(4) if (next hop $==$ false) then

(5) check for current attraction value

(6) if (Conditions (9) and (15)) == true then

(7) perform re-announcements and re-route

(8) else

(9) check for next node which is not selected earlier

(10) mark the node and check for degree of connectivity

(11) if (slots available $==$ true) then

(12) connect and proceed

(13) exit(0)

(14) else

(15) select node with next in the order of attraction

(16) reduce the number of outgoing and incoming connections

(17) check for steps (5)-(11)

(18) end if

(19) end if

(20) end if

(21) end while

Algorithm 4: Route rehabilitation and maintenance.

TABLE 1: Parameter configurations.

\begin{tabular}{lcc}
\hline Parameter & Value & Description \\
\hline$A$ & $50 \times 50$ sq. m. & Region of interest \\
$\lambda=m$ & $100-300$ & Sensor distribution \\
$C_{h}$ & 3 & Channels \\
$M_{s}$ & 1024 & Messages over $C_{h}$ \\
$E_{\mathrm{TX}}$ & $0.34 \mathrm{~J}$ & Transmitter energy \\
$E_{P}$ & $0.16 \mathrm{~J}$ & consumption \\
$\mathrm{EU}_{0}$ sensors & $150 \mathrm{~J}$ & Receiver processing energy \\
$\mathrm{EU} \mathrm{U}_{0} \mathrm{UAVs}$ & $2000 \mathrm{~J}$ & Initial energy available \\
$S$ & $3-5$ & Initial energy available \\
$n$ & 1 per Segment & Segments \\
$r$ & $10 \mathrm{~m}$ & Aerial vehicles \\
$\rho$ & 4 & Sensors' radio range \\
$\mu_{a}$ & $256 \mathrm{bps}$ & Pathloss for UAV model \\
$\mu_{s}$ & $1024 \mathrm{Bytes}$ & Packet arrival rate \\
$\max \left(C_{p}\right)$ & $1.2 \mathrm{Gbps}$ & Packet size \\
\hline
\end{tabular}

measure of efficient data dissemination. A higher packet delivery ratio at an optimal utilization of the energy indicates the effectiveness of the proposed model.

(iv) Delays: with more numbers of intermediate hops and rerouting, the possibility of delays increases. An optimal model does not allow delays to go beyond a certain value above which the network may fail or may lead to overconsumption of energy resources. Delays are measured as timely response with respect to the offered load. (v) Lifetime: as the network operates, energy depletes and at last whole energy of the network gets used. A network with better lifetime operates for longer duration. Thus, analyzing the extent up to which the network operates is marked as the lifetime of the network. Lifetime is computed on the basis of the iterations required to transmit data from the sensor to the base station. This metric is observed with respect to the percentage energy consumed.

(vi) Coverage: this metric is defined with respect to [14] which accounts for the total area supported using the active sensors with respect to the total area over which the sensors are deployed.

(vii) Excessive Iterations per Segment: in a network operating over sensors with limited amount of energy, possibility of requirement of new routes is high, and thus requires more iterations for path selection. Excessive iterations lead to excessive wastage of energy. Also, with iterations at peak, probability of routing loop also increases.

The proposed approach was evaluated against the above defined metrics in comparison with the EEGA [17], ERIDSR [14], and I-ERIDSR [16] approaches. Two independent parameters taken for analysis were energy consumption and active sensors. All the three models were evaluated for the same parameters over which the proposed approach was configured. Results show that the proposed model is capable of finding optimal routes without any loops and is capable of offering better traffic rates in comparison with the existing approaches. The proposed approach provides 5.5\%, 9.6\%, and $13.6 \%$ better throughput than the EEGA, I-ERIDSR, and ERIDSR, respectively, as shown in Figure 6. 


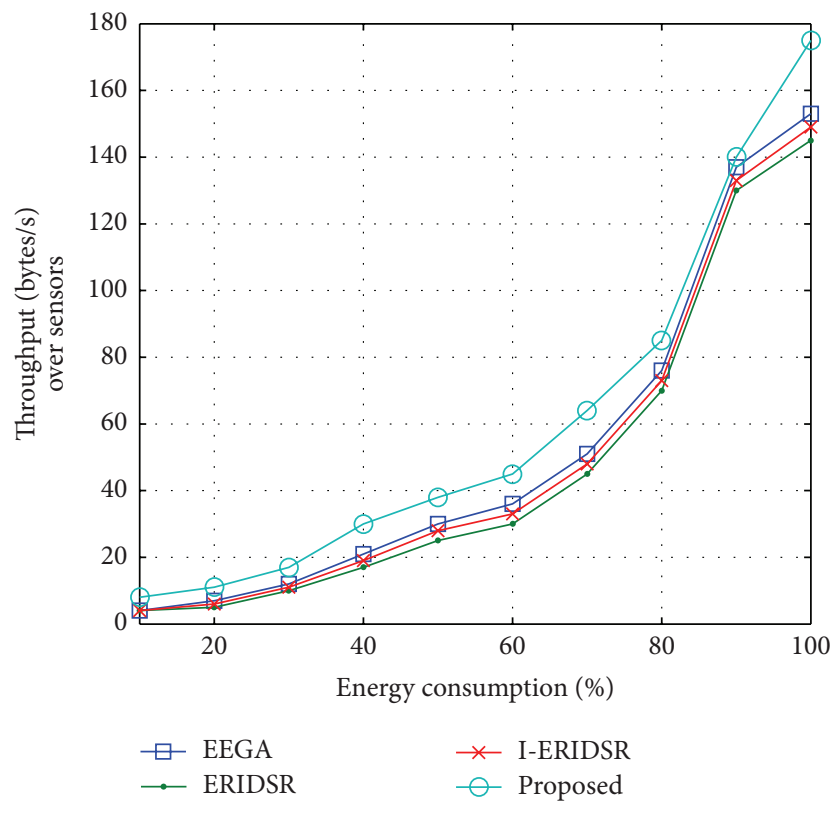

FIgURE 6: Throughput versus energy consumption.

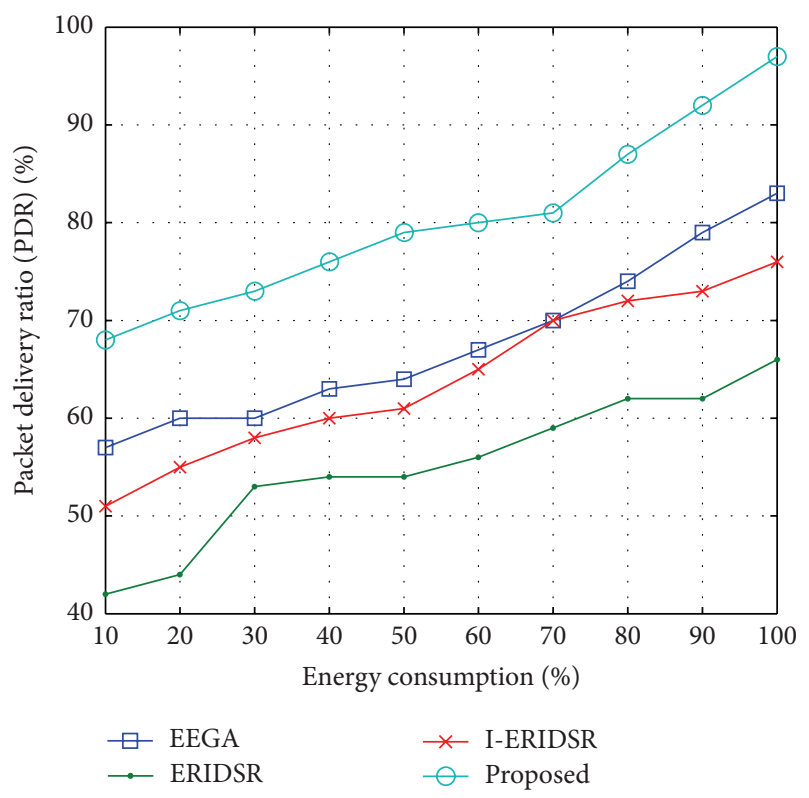

FIGURE 7: PDR versus energy consumption.

An efficient approach accounts for accurate delivery of data. This measurement is defined in terms of packet delivery ratio (PDR). With lesser number of iterations and better connectivity between the sensors and the UAVs, the proposed approach provides $17.2 \%, 18.01 \%$, and $31.5 \%$ better PDR than the EEGA, I-ERIDSR, and ERIDSR, respectively, as shown in Figure 7 . The more the number of iterations, the more the requirement of intermediate nodes; thus, excess is the energy consumption and vice versa. In contrast with the energy consumption, mean hop requirement in transmission of data between the sensors and the base station via UAVs increases. Results show that the proposed approach requires $51 \%, 61.5 \%$,

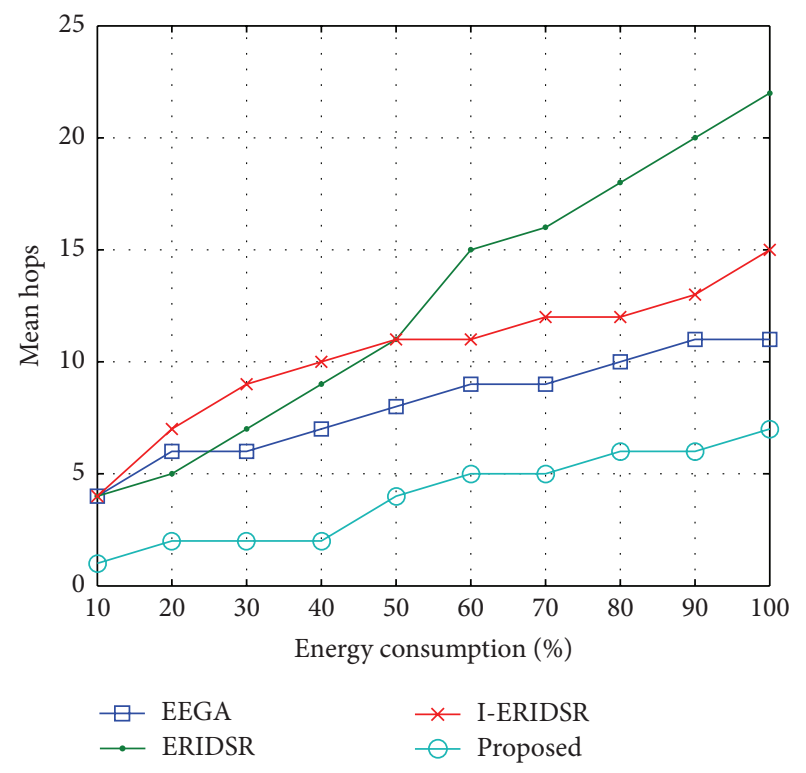

FIGURE 8: Mean hops versus energy consumption.

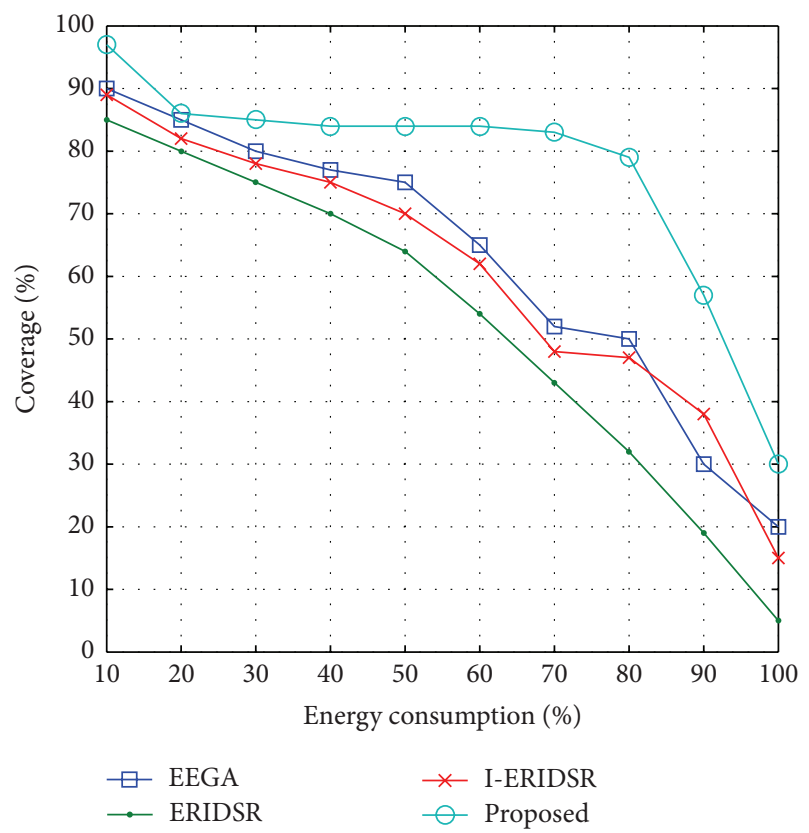

Figure 9: Coverage versus energy consumption.

and $68.5 \%$ less intermediate hops than the EEGA, I-ERIDSR, and ERIDSR, respectively, as shown in Figure 8.

With more consumption of energy, probability of sensors going into dead state increases, which results in lesser number of active sensors, thus, decreasing the percentage coverage of area. Analysis shows that the proposed approach provides $18.8 \%, 21.4 \%$, and $31.4 \%$ better coverage than the EEGA, IERIDSR, and ERIDSR, respectively, as shown in Figure 9. In a network, the delay provides a better understanding of network operations. A network with delay beyond a limiting value can lead to a dead state. Delay cannot be completely removed from the network; however, delay below a certain 


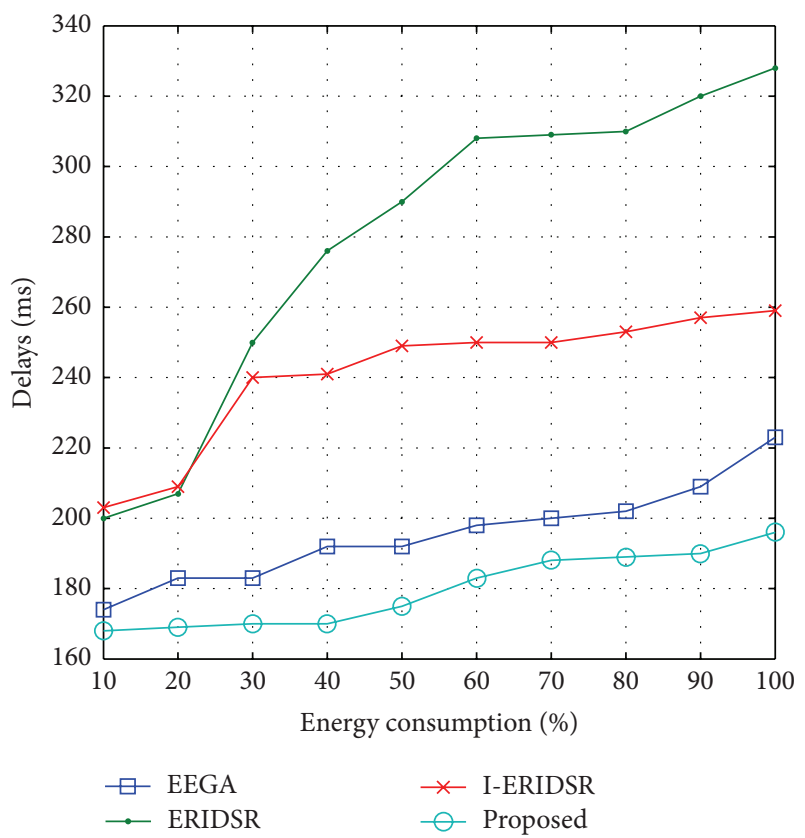

FIGURE 10: Delays versus energy consumption.

range does not affect the network performance. Analysis shows that the delay in the proposed model is quite low and does not affect the network performance. Results presented in Figure 10 show that the proposed approach causes 10\%, 27.5\%, and $41.5 \%$ less delay than the EEGA, I-ERIDSR, and ERIDSR, respectively. The more numbers of intermediate hops lead to the problem of routing loop; further, it also increases the requirement of excessive iterations. These excessive iterations lead to the overconsumption of energy resources that finally decrease the network operability. Results presented in Figure 11 show that the proposed approach requires $60.6 \%$, $70.4 \%$, and $83.11 \%$ lesser iterations than the EEGA, I-ERIDSR, and ERIDSR, respectively.

A detailed analysis of the proposed approach over energy model suggests that the proposed approach provides $19.19 \%$, $31.3 \%$, and $50.1 \%$ better lifetime, in terms of the network iterations, than the EEGA, I-ERIDSR, and ERIDSR, respectively, as shown in Figure 12. The proposed approach does not cause any routing loop issues, thus allowing the network to operate without any hindrance. This continuation leads to increased lifetime and improved connectivity. Active sensors also affect the performance of the network to a large extent. The more the number of active sensors, the better the network performance. Analyses were also traced for PDR and energy consumption with respect to the percentage of active sensors. With increasing number of sensors, the proposed approach provides a $5.7 \%, 12.01 \%$, and $25.7 \%$ better PDR than the EEGA, I-ERIDSR, and ERIDSR, respectively, as shown in Figure 13 and consumes 21.8\%, 24.14\%, and 34.3\% less energy in contrast to EEGA, I-ERIDSR, and ERIDSR, respectively, as shown in Figure 14.

Tables 2 and 3 present the detailed percentage improvement by the proposed model in comparison with EEGA, IERIDSR, and ERIDSR over energy consumption and active

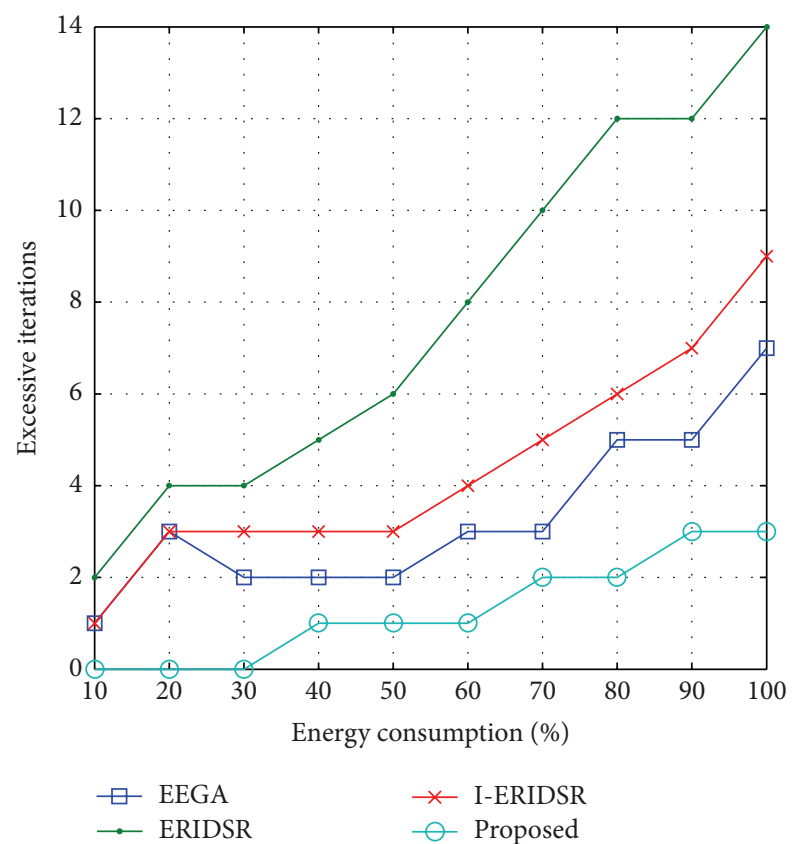

Figure 11: Excessive iterations versus energy consumption.

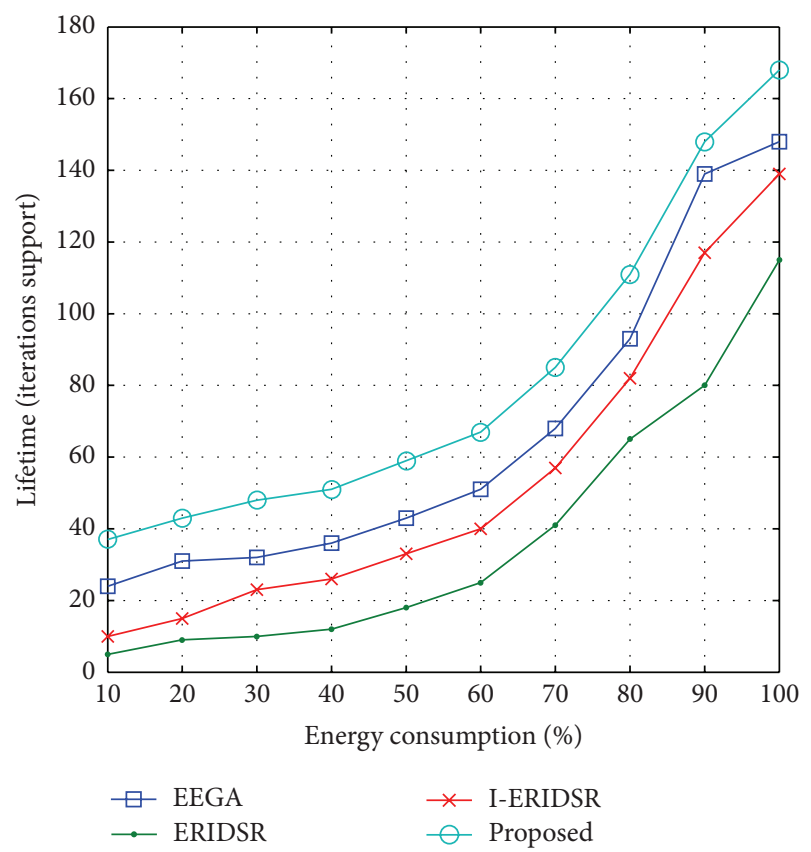

FIGURE 12: Lifetime versus energy consumption.

sensors, respectively; and Table 4 gives the overall comparison between the EEGA, I-ERIDSR, ERIDSR, and the proposed approach.

\section{Conclusion}

Data dissemination in wireless sensor networks (WSNs) is a crucial task that consumes a lot of network resources. Further, improper relaying of data can lead to a problem of routing 


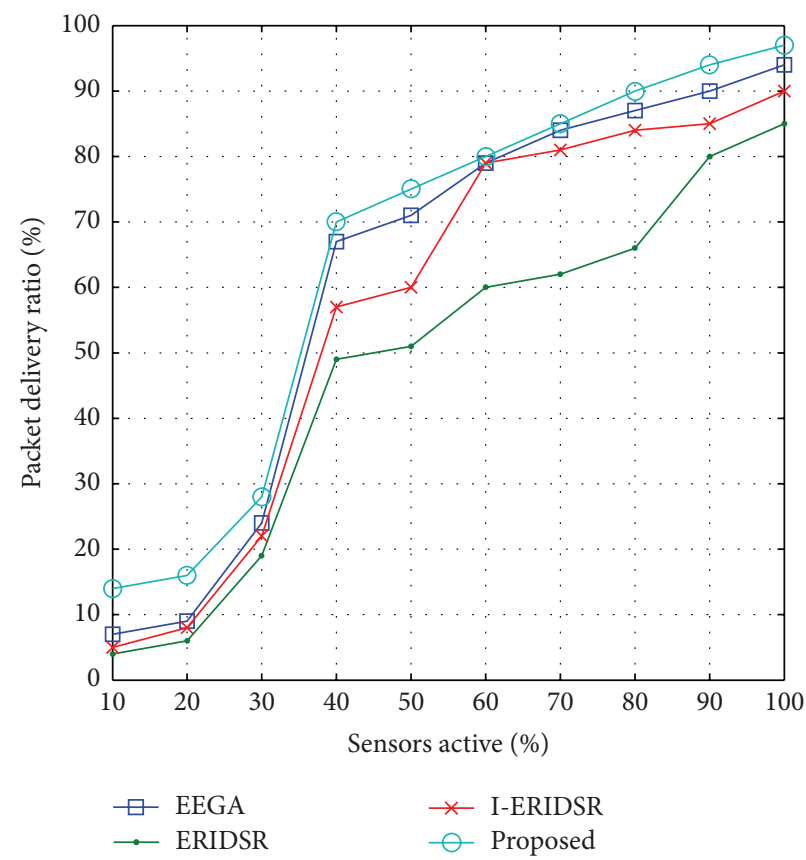

FIGURE 13: PDR versus active sensors.

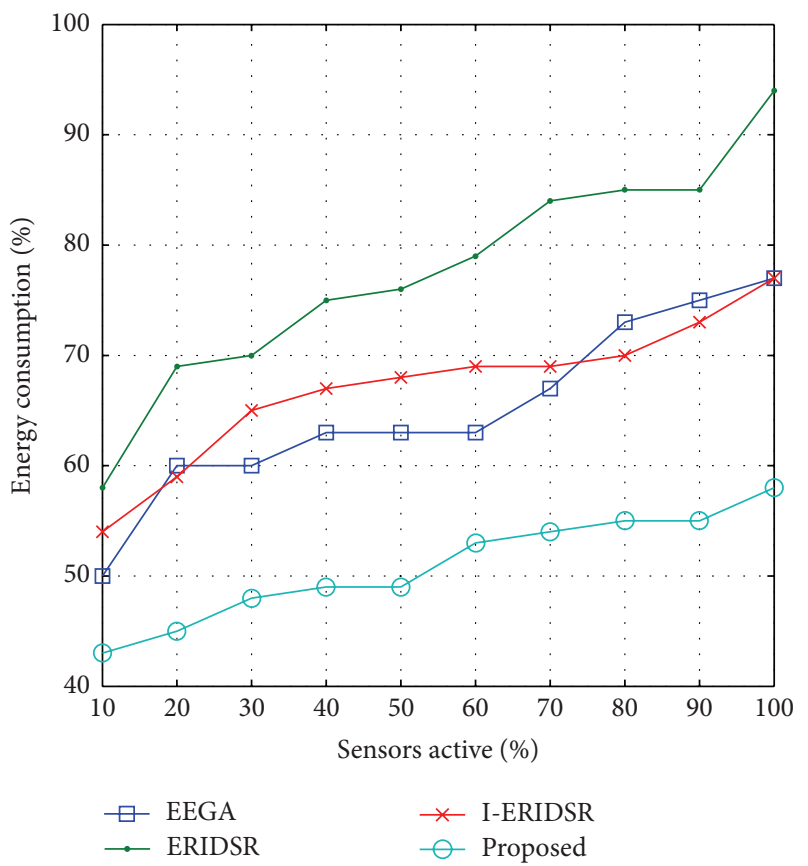

FIGURE 14: Energy consumption versus active sensors.

loop that causes wastage of network resources, and leads the network into a dead state. A novel approach is proposed in this paper which combines the WSNs with the unmanned aerial vehicles (UAVs) to efficiently disseminate the data between the sensors and the base station. UAVs play a pivotal role in the proposed model and provide support as a manger node. The proposed model uses the attraction properties of fire fly optimization algorithm for route discovery and
TABLE 2: Average percentage improvement by the proposed approach in comparison with EEGA, I-ERIDSR, and ERIDSR with respect to energy consumption.

\begin{tabular}{lccc}
\hline Metrics & EEGA & I-ERIDSR & ERIDSR \\
\hline Packet delivery ratio (PDR) & $17.2 \%$ & $18.01 \%$ & $31.5 \%$ \\
Throughput & $5.5 \%$ & $13.6 \%$ & $9.6 \%$ \\
Mean hops & $51 \%$ & $61.5 \%$ & $68.5 \%$ \\
Coverage & $18.8 \%$ & $21.4 \%$ & $31.4 \%$ \\
Delays & $10 \%$ & $27.5 \%$ & $41.5 \%$ \\
Excessive iterations & $60.6 \%$ & $70.4 \%$ & $83.11 \%$ \\
Lifetime & $19.19 \%$ & $31.3 \%$ & $50.1 \%$ \\
\hline
\end{tabular}

TABLE 3: Average percentage improvement by the proposed approach in comparison with EEGA, I-ERIDSR, and ERIDSR with respect to active sensors.

\begin{tabular}{lccc}
\hline Metrics & EEGA & I-ERIDSR & ERIDSR \\
\hline Packet delivery ratio (PDR) & $5.7 \%$ & $10.01 \%$ & $25.7 \%$ \\
Energy consumption & $21.8 \%$ & $24.14 \%$ & $34.3 \%$ \\
\hline
\end{tabular}

maintenance. The proposed approach is tested in comparison with the EEGA, ERIDSR, and I-ERIDSR approaches over various metrics. Analyses prove that the proposed approach is capable of providing better lifetime and loop-free routing over UAV coordinated WSNs with lesser delays and improved coverage.

\section{Competing Interests}

The authors declare that there are no competing interests regarding the publication of this paper. 
TABLE 4: Overall comparison between EEGA, I-ERIDSR, ERIDSR, and the proposed approach.

\begin{tabular}{lccccccc}
\hline Approach & Packet delivery ratio (PDR) & Throughput & Mean hops & Coverage & Delays & Excessive iterations & Lifetime \\
\hline Proposed & Highest & Highest & Least & Highest & Least & Least & Highest \\
EEGA & High & High & Low & High & Low & Low & High \\
I-ERIDSR & Medium & Medium & Medium & Medium & Medium & Medium & Medium \\
ERIDSR & Low & Low & High & Low & High & High & Low \\
\hline
\end{tabular}

\section{Acknowledgments}

This work was partially supported by the Soonchunhyang University Research Fund and also supported in part by the Basic Science Research Program through the National Research Foundation of Korea (NRF) funded by the Ministry of Science, ICT and Future Planning (2014R1A1A1005915).

\section{References}

[1] V. Sharma and R. Kumar, "A cooperative network framework for multi-UAV guided ground ad hoc networks," Journal of Intelligent \& Robotic Systems, vol. 77, no. 3-4, pp. 629-652, 2015.

[2] S. Berrahal, J.-H. Kim, S. Rekhis, N. Boudriga, D. Wilkins, and J. Acevedo, "Unmanned aircraft vehicle assisted WSN-based border surveillance," in Proceedings of the 23rd International Conference on Software, Telecommunications and Computer Networks (SoftCOM '15), pp. 132-137, IEEE, Split, Croatia, September 2015.

[3] V. Sharma and R. Kumar, "An opportunistic cross layer design for efficient service dissemination over flying ad hoc networks (FANETs)," in Proceedings of the 2nd International Conference on Electronics and Communication Systems (ICECS '15), pp. 1551-1557, Coimbatore, India, February 2015.

[4] V. Sharma, R. Kumar, and P. S. Rana, "Self-healing neural model for stabilization against failures over networked UAVs," IEEE Communications Letters, vol. 19, no. 11, pp. 2013-2016, 2015.

[5] I. F. Akyildiz, W. Su, Y. Sankarasubramaniam, and E. Cayirci, "Wireless sensor networks: a survey," Computer Networks, vol. 38, no. 4, pp. 393-422, 2002.

[6] K. Akkaya and M. Younis, "A survey on routing protocols for wireless sensor networks," Ad Hoc Networks, vol. 3, no. 3, pp. 325-349, 2005.

[7] A. T. Erman, L. V. Hoesel, P. Havinga, and J. Wu, "Enabling mobility in heterogeneous wireless sensor networks cooperating with UAV sor mission-critical management," IEEE Wireless Communications, vol. 15, no. 6, pp. 38-46, 2008.

[8] C. Gu and Q. Zhu, "An energy-aware routing protocol for mobile ad hoc networks based on route energy comprehensive index," Wireless Personal Communications, vol. 79, no. 2, pp. 1557-1570, 2014.

[9] G. Anastasi, M. Conti, M. Di Francesco, and A. Passarella, "Energy conservation in wireless sensor networks: a survey," Ad Hoc Networks, vol. 7, no. 3, pp. 537-568, 2009.

[10] E. Basha, M. Eiskamp, J. Johnson, and C. Detweiler, "UAV recharging opportunities and policies for sensor networks," International Journal of Distributed Sensor Networks, vol. 2015, Article ID 824260, 10 pages, 2015.

[11] H. H. Choi, H. Choi, M. Choi, T. Shon, and B. Park, "An obstacle avoidance scheme maintaining connectivity for microunmanned aerial vehicles," International Journal of Distributed Sensor Networks, vol. 2014, Article ID 920534, 11 pages, 2014.
[12] X.-S. Yang, "Multiobjective firefly algorithm for continuous optimization," Engineering with Computers, vol. 29, no. 2, pp. 175-184, 2013.

[13] B. S. Faiçal, F. G. Costa, G. Pessin et al., "The use of unmanned aerial vehicles and wireless sensor networks for spraying pesticides," Journal of Systems Architecture, vol. 60, no. 4, pp. 393404, 2014.

[14] C.-E. Weng and T.-W. Lai, "An energy-efficient routing algorithm based on relative identification and direction for wireless sensor networks," Wireless Personal Communications, vol. 69, no. 1, pp. 253-268, 2013.

[15] H. Oh, H. Bahn, and K.-J. Chae, "An energy-efficient sensor routing scheme for home automation networks," IEEE Transactions on Consumer Electronics, vol. 51, no. 3, pp. 836-839, 2005.

[16] G. Wei, S. Yan, and S. Fan, "Routing algorithm based on area division management of node in wireless sensor networks," TELKOMNIKA (Telecommunication Computing Electronics and Control), vol. 13, no. 4, pp. 1214-1224, 2015.

[17] H. Safa, M. Moussa, and H. Artail, "An energy efficient genetic algorithm based approach for sensor-to-sink binding in multisink wireless sensor networks," Wireless Networks, vol. 20, no. 2, pp. 177-196, 2014.

[18] S. Zhang, A. Yan, and T. Ma, "Energy-balanced routing for maximizing network lifetime in wirelessHART," International Journal of Distributed Sensor Networks, vol. 2013, Article ID 173185, 7 pages, 2013.

[19] E. P. De Freitas, T. Heimfarth, A. M. Ferreira, C. E. Pereira, F. R. Wagner, and T. Larsson, "Decentralized task distribution among cooperative UAVs in surveillance systems applications," in Proceedings of the 7th International Conference on Wireless On-Demand Network Systems and Services (WONS '10), pp. 121128, IEEE, Kranjska Gora, Slovenia, February 2010.

[20] M. Abdelhakim, Z. Fang, J. Ren, and T. Li, "Hybrid mobile access coordinated wireless sensor networks-design and analysis," in Proceedings of the IEEE China Summit and International Conference on Signal and Information Processing (ChinaSIP '15), pp. 453-457, Chengdu, China, July 2015.

[21] S. Valuiskyi, A. Lysenko, T. Pryshchepa, and S. Chumachenko, "The problem of finding a rational topology of wireless sensor networks using UAVs," in Proceedings of the 2nd International Scientific-Practical Conference Problems of Infocommunications Science and Technology (PIC S\&T '15), pp. 213-215, IEEE, Kharkiv, Ukraine, October 2015.

[22] Y. Li, H. Chen, S. Mo, and H. Liu, "Optimal query-driven data forwarding for delay-sensitive wireless sensor networks," Wireless Personal Communications, vol. 77, no. 1, pp. 41-62, 2014. 

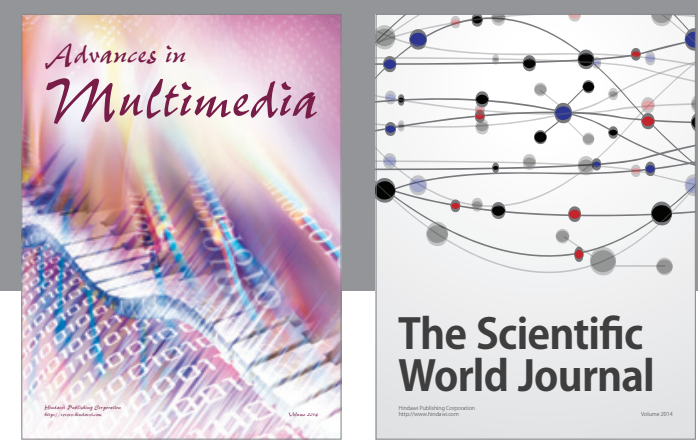

The Scientific World Journal
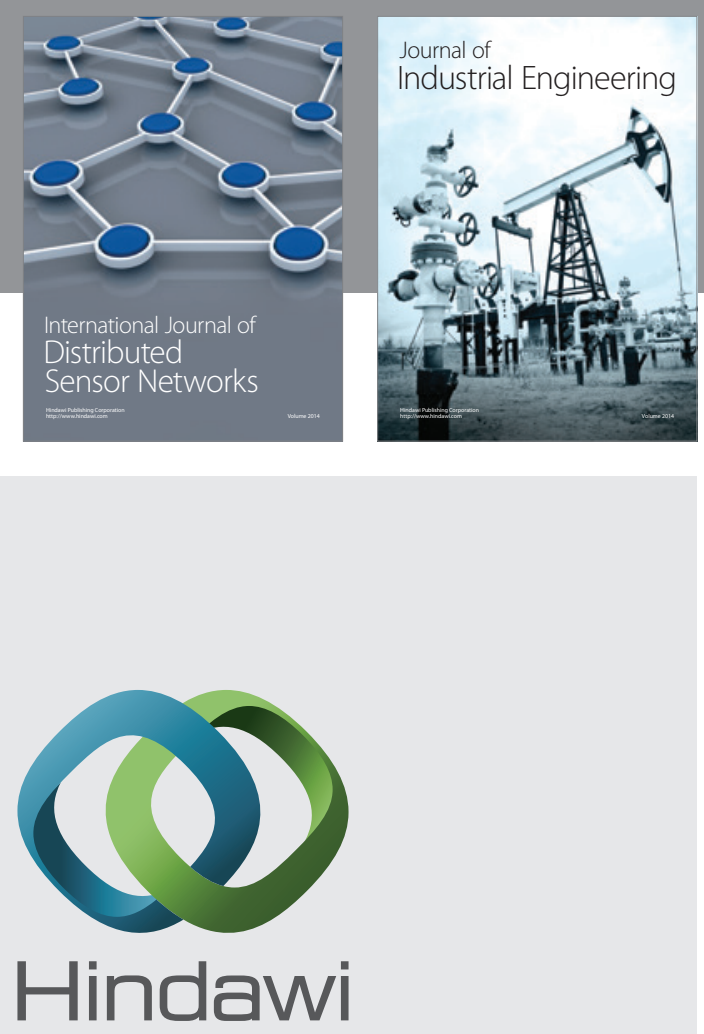

Submit your manuscripts at

http://www.hindawi.com

\section{Computer Networks} and Communications
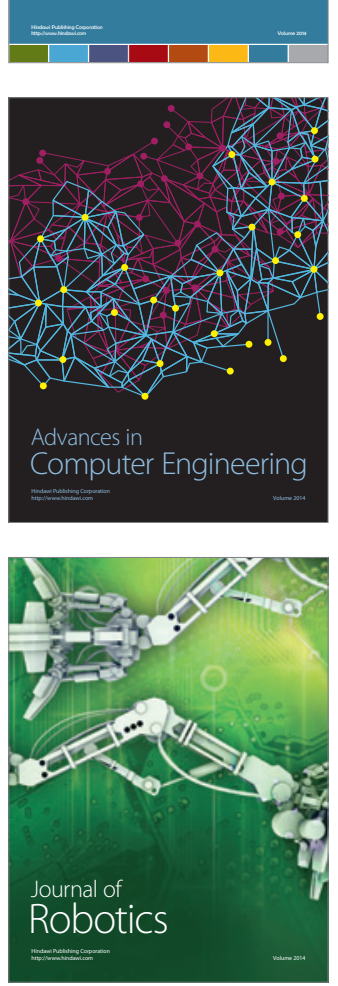
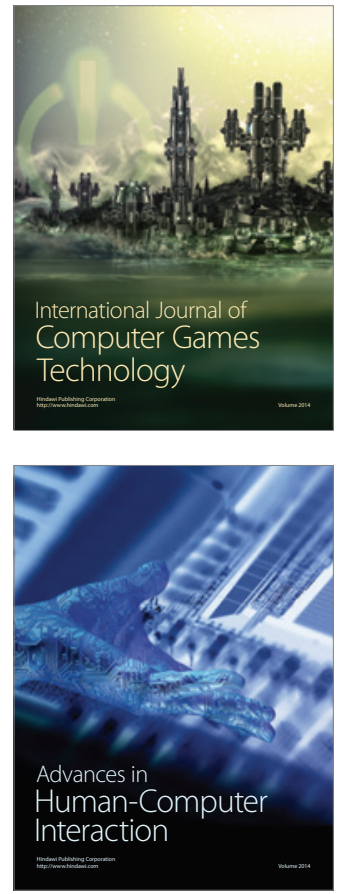
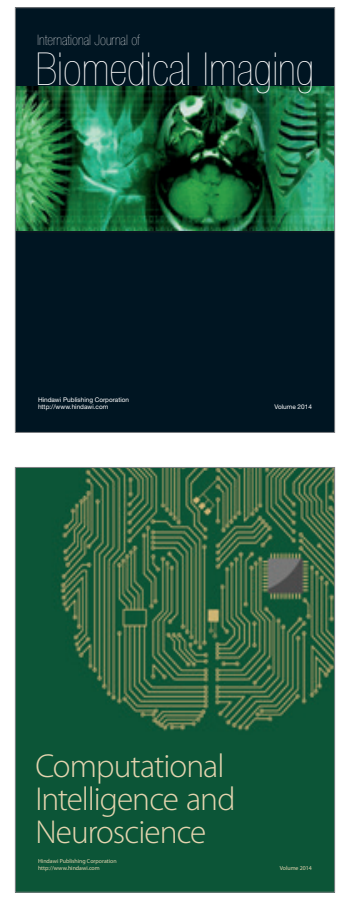
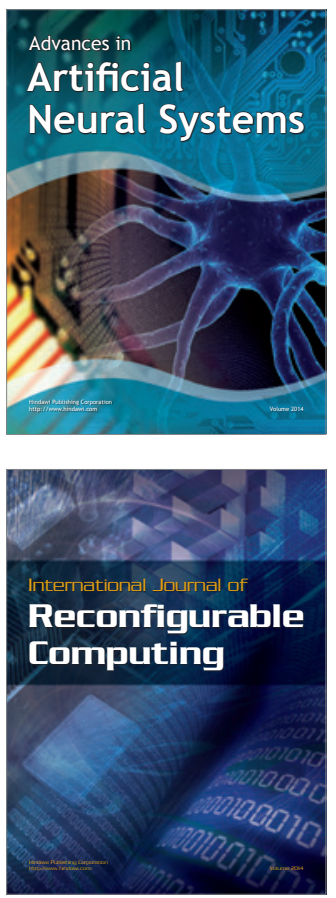
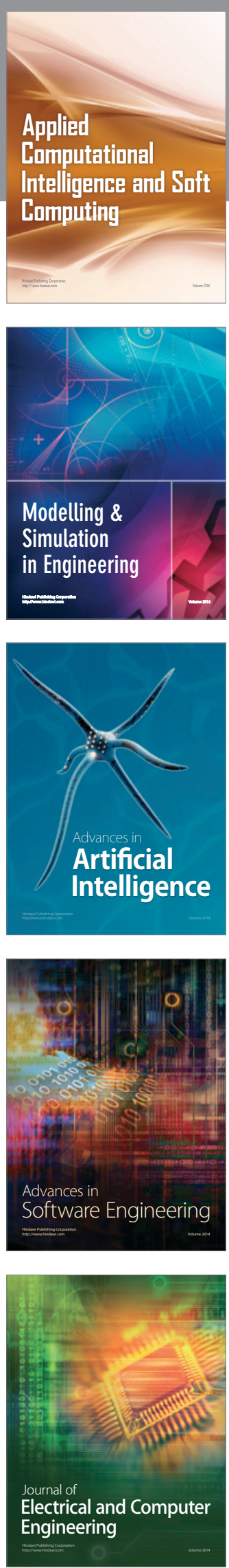